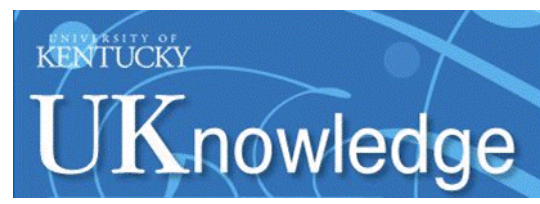

University of Kentucky

UKnowledge

6-1-2017

\title{
Neurophysiological Capacity in a Working Memory Task Differentiates Dependent from Nondependent Heavy Drinkers and Controls
}

\author{
Michael J. Wesley \\ University of Kentucky, michael.wesley@uky.edu \\ Joshua A. Lile \\ University of Kentucky, jalile2@uky.edu \\ Mark T. Fillmore \\ University of Kentucky, fillmore@uky.edu \\ Linda J. Porrino \\ Wake Forest University
}

Follow this and additional works at: https://uknowledge.uky.edu/behavsci_facpub

Part of the Behavior and Behavior Mechanisms Commons, Psychology Commons, and the Substance Abuse and Addiction Commons

Right click to open a feedback form in a new tab to let us know how this document benefits you.

\section{Repository Citation}

Wesley, Michael J.; Lile, Joshua A.; Fillmore, Mark T.; and Porrino, Linda J., "Neurophysiological Capacity in a Working Memory Task Differentiates Dependent from Nondependent Heavy Drinkers and Controls" (2017). Behavioral Science Faculty Publications. 40.

https://uknowledge.uky.edu/behavsci_facpub/40

This Article is brought to you for free and open access by the Behavioral Science at UKnowledge. It has been accepted for inclusion in Behavioral Science Faculty Publications by an authorized administrator of UKnowledge. For more information, please contact UKnowledge@lsv.uky.edu. 
Neurophysiological Capacity in a Working Memory Task Differentiates Dependent from Nondependent Heavy Drinkers and Controls

Digital Object Identifier (DOI)

https://doi.org/10.1016/j.drugalcdep.2017.01.029

Notes/Citation Information

Published in Drug and Alcohol Dependence, v. 175, p. 24-35.

(C) 2017 Elsevier B.V. All rights reserved.

This manuscript version is made available under the CC-BY-NC-ND 4.0 license

https://creativecommons.org/licenses/by-nc-nd/4.0/.

The document available for download is the author's post-peer-review final draft of the article. 
Drug Alcohol Depend. 2017 June 01; 175: 24-35. doi:10.1016/j.drugalcdep.2017.01.029.

\title{
Neurophysiological capacity in a working memory task differentiates dependent from nondependent heavy drinkers and controls
}

\author{
Michael J. Wesley ${ }^{1}$, Joshua A. Lile ${ }^{1,2}$, Mark T. Fillmore ${ }^{2}$, and Linda J. Porrino ${ }^{3}$ \\ ${ }^{1}$ Department of Behavioral Science, University of Kentucky College of Medicine, Lexington \\ Kentucky, USA \\ ${ }^{2}$ Department of Psychology, University of Kentucky College of Arts and Sciences, Lexington \\ Kentucky, USA \\ ${ }^{3}$ Department of Physiology and Pharmacology, Wake Forest University School of Medicine, \\ Winston-Salem, North Carolina, USA
}

\begin{abstract}
Background-Determining the brain-behavior profiles that differentiate heavy drinkers who are and are not alcohol dependent will inform treatment efforts. Working memory is linked to substance use disorders and can serve as a representation of the demand placed on the neurophysiology associated with cognitive control.
\end{abstract}

Methods-Behavior and brain activity (via fMRI) were recorded during an N-Back working memory task in controls (CTRL), nondependent heavy drinkers (A-ND) and dependent heavy drinkers (A-D). Typical and novel step-wise analyses examined profiles of working memory load and increasing task demand, respectively.

Results-Performance was significantly decreased in A-D during high working memory load (2Back), compared to CTRL and A-ND. Analysis of brain activity during high load (0-Back vs. 2Back) showed greater responses in the dorsal lateral and medial prefrontal cortices of A-D than CTRL, suggesting increased but failed compensation. The step-wise analysis revealed that the transition to Low Demand (0-Back to 1-Back) was associated with robust increases and decreases in cognitive control and default-mode brain regions, respectively, in A-D and A-ND but not

\footnotetext{
To whom correspondence should be addressed: Michael J. Wesley, Ph.D., Medical Behavioral Science Building, Lexington, Kentucky, USA, Phone: 859-323-1332, Fax: 859-323-5350, michael.wesley@uky.edu.

Publisher's Disclaimer: This is a PDF file of an unedited manuscript that has been accepted for publication. As a service to our customers we are providing this early version of the manuscript. The manuscript will undergo copyediting, typesetting, and review of the resulting proof before it is published in its final citable form. Please note that during the production process errors may be discovered which could affect the content, and all legal disclaimers that apply to the journal pertain.

Author Disclosures: None

Conflicts of interest: none

Contributors: MJ Wesley assisted in theoretical framework, study design, data collection/analysis and manuscript preparation. JA Lile assisted in data analysis and manuscript preparation. MT Fillmore assisted in theoretical framework and manuscript preparation. LJ Porrino provided laboratory resources and assisted in study design, theoretical framework, data analysis and manuscript preparation. All authors have approved the article for submission.
} 
CTRL. The transition to High Demand (1-Back to 2-Back) resulted in additional engagement of these networks in A-ND and CTRL, but not A-D.

Conclusion-Heavy drinkers engaged working memory neural networks at lower demand than controls. As demand increased, nondependent heavy drinkers maintained control performance but relied on additional neurophysiological resources, and dependent heavy drinkers did not display further resource engagement and had poorer performance. These results support targeting these brain areas for treatment interventions.

\section{Keywords}

Addiction; Alcohol; Working Memory; fMRI; Executive Function

\subsection{Introduction}

Not all heavy drinkers develop alcohol use disorder, despite having similar alcohol use patterns. Identifying brain-behavior patterns that differentiate heavy drinkers who develop alcohol use disorder from those who do not will provide valuable information about the individual differences associated with this disorder, thereby informing treatment efforts. A common approach used to characterize brain-behavior abnormalities associated with mental health problems like alcohol use disorder is to compare individuals with a particular condition to controls using experimental tasks designed to measure specific neural processes and associated behavioral output within a neuroimaging environment. In this way, challenging brain activity and behavior during task performance can uncover deficiencies and/or compensatory mechanisms. In particular, the cognitive control of behavior and interconnected processes such as working memory have been a research focus due to their essential roles in normal psychosocial functioning. For example, studies using working memory tasks and neuroimaging techniques have demonstrated working memory deficits are linked to altered brain activity in various mental health disorders including, but not limited to, schizophrenia (Jansma et al., 2004), pathological dissociation (Elzinga et al., 2007) and bipolar disorder (Cremaschi et al., 2013).

Abnormal working memory has also been identified as a critical concern in substance use disorders, having been demonstrated in individuals dependent on alcohol (Pitel et al., 2007) and various other classes of drugs including opioids (Vo et al., 2014), cannabis (Vo et al., 2014), cocaine (Albein-Urios et al., 2012) and methamphetamine (Duarte et al., 2012). That working memory deficits are found across problematic users of alcohol and other drugs is perhaps not surprising considering that impaired control over the use of these substances is a characteristic of substance use disorders and this behavioral control relies, in part, on working memory (Hofmann et al., 2008). More specifically, control over the decision to use alcohol and other drugs requires working memory to integrate information related to previous consequences of substance use and current contingencies surrounding the decision to use (Weber and Johnson, 2009). Reduced working memory capacity in these individuals might be more easily overloaded during the evaluation of this information, resulting in impulsive, maladaptive substance use decisions in the present and for the future (Wesley and Bickel, 2014; Wesley et al., 2014). Indeed, several theories of impulsivity as a factor in drug abuse and in externalizing disorders, such as ADHD, posit a bi-directional relationship 
between working memory and inhibitory control (Barkley, 1997; Fillmore, 2003; Finn, 2002). Consistent with this idea, studies have shown that low working memory ability is associated with greater trait impulsivity (e.g., Gunn and Finn, 2013). Experimental manipulation of working memory load has revealed that increasing load to a capacity limit increases impulsive performance on a decision-making task (Hinson et al., 2003). Also, research on the acute effects of alcohol on inhibitory control showed that the disinhibiting effects of the drug are, in part, due to the alcohol-induced reductions in information processing capacity (Fillmore and Van Selst, 2002). Taken together, these lines of evidence provide corroborating evidence for the important link between working memory and undercontrolled behavior.

A substantial amount of clinical research has characterized the neurocircuitry underlying cognitive control and working memory, as well as the network-level changes that occur as these processes are engaged. A recent meta-analysis (Niendam et al., 2012) of 193 functional magnetic resonance imaging (fMRI) studies indicated that the primary brain regions involved in the cognitive control of behavior, including prefrontal cortex regions, such as the dorsal lateral prefrontal cortex (DLPFC) and the anterior cingulate cortex (ACC), as well as the superior and inferior parietal cortices (sPar), precuneus and precentral gyrus. In general, these areas are part of an integrated dorsal processing stream involved in overlapping cognitive functions (Wesley and Bickel, 2014) that is engaged in response to external demand. For example, engagement of the DLPFC, superior parietal cortices, ACC, striatum, thalamus and insula has been detected by fMRI during working memory tasks, in line with the performance requirements of those tasks, such as match mismatch detection and response inhibition (reviewed in Wilcox et al., 2014). By contrast, when external demand is not present and the brain is considered to be in the default mode of functioning, activity is greater in brain areas such as the ventral medial prefrontal cortex (vmPFC) and posterior cingulate cortex (PCC) (Raichle and Snyder, 2007; Wang and Li, 2013). As external demand is imposed, activity typically decreases in the default-mode network and increases in cognitive control networks, consistent with the heuristics of a free energy framework for biological systems (Friston, 2009, 2010; Friston et al., 2006).

Altered brain activity has been demonstrated using fMRI during the performance of working memory tasks in individuals with substance use disorders. By way of introduction to the present study, the following overview focuses on research in adults with alcohol use disorder that used N-Back type tasks, which are well-established working memory tasks that have been adapted for use in a neuroimaging environment. While performing a 2-Back spatial working memory task, individuals with alcohol use disorder displayed reduced bilateral DLPFC activation compared to control participants when performing the task at equivalent level (Pfefferbaum et al., 2001). In another study (Tapert et al., 2001), emerging adult (i.e., 18-25 years old) women with alcohol use disorder and matched controls completed a task in which they were instructed to respond when an abstract line drawing appeared in a location that had been previously occupied. Participants with alcohol use disorder were less accurate on the task and had less activity in the right superior and inferior parietal, right middle frontal, right postcentral and left superior frontal cortex compared to controls. In a study that used a non-spatial 2-Back task, reduced activity was observed in the bilateral frontal and pre-central cortex and left superior temporal and parietal cortices of individuals with alcohol 
use disorder compared to social drinkers (Park et al., 2011). Importantly, these impairments in working memory performance and altered activity in associated brain areas appear to be clinically significant. For example, individuals with alcohol use disorder who exhibited greater bilateral rostral and ventral lateral, prefrontal cortex activity given equivalent performance of a 2-Back task were more likely to remain abstinent through a 7 month posttreatment period (Charlet et al., 2014).

A limitation of prior studies that have evaluated working memory performance and associated brain activity in individuals with alcohol use disorder is that a control group with similar alcohol use history has not been included to differentiate between alcohol exposure and problematic behaviors. Furthermore, those studies have not considered dynamic changes in brain activity and/or recruitment of other regions as a step-wise function of neurophysiological demand. Understanding how brain function changes in response to increasing task demand, however, could provide new insights into the neural resources (or lack thereof) available to meet the external constraints placed on the cognitive control processes needed for successful abstinence and/or recovery. The present study sought to extend previous work by comparing working memory performance and associated brain activity in individuals who met criteria for alcohol use disorder (heavy drinkers who were dependent; A-D) to a group with a comparable alcohol use history (heavy drinkers who were nondependent; A-ND), as well as a group of individuals who reported non-problematic alcohol use (CTRL). Further, this study included zero- (0-Back), low- (1-Back) and high- (2Back) working memory load conditions. In addition to typical analyses examining brain activity during low (0-Back vs. 1-Back) and high (0-Back vs. 2- Back) working memory load conditions, the current study used a step-wise transitional approach to determine the concurrent increases and decreases in brain activity associated with transitioning to neurophysiological states with low (1-Back $>0$-Back and 1-Back $<0$-Back) and high (2Back > 1-Back and 2-Back < 1-Back) external demand on neurophysiological resources. In this way, we sought to identify potential differences in the neurophysiological capacity associated with a critical cognitive process in individuals who vary in their alcohol use history and expression of problem drinking behaviors. To the extent that neurobehavioral impairments are associated with alcohol dependence and not just heavy alcohol consumption, we predicted reduced performance and brain activity in heavy drinkers who were dependent relative to the nondependent heavy drinkers.

\subsection{Methods}

\subsection{Participants}

The Wake Forest School of Medicine Institutional Review Board approved the study protocol. Potential participants responded to local media advertisements by phone and an initial screen was performed to determine study eligibility. Individuals reporting illicit drug use, diseases of the central nervous system, head trauma, current use of psychotropic medications, or any condition that prohibited entry into an MRI scanner were ineligible. Individuals who passed this initial screening were invited to the laboratory for additional screening and were asked to abstain from alcohol use for a minimum of twelve hours prior to their visit. 
Once arriving at the laboratory, expired air samples were tested for alcohol (Intoxilyzer SD-5; CMI Inc., Owensboro, KY) and urine samples were tested for illicit drugs (Multipanel Urine Screen; Innovacon, Inc, San Diego, CA) and pregnancy (QuickVue urine HCG test; Quidel Inc, San Diego, CA). Individuals who tested positive for alcohol, other drugs (i.e., marijuana, cocaine, amphetamines, opiates and benzodiazepines) or pregnancy were excluded from participation. To evaluate alcohol use history, psychiatric conditions, and intelligence, potential participants completed the Alcohol Use Disorders Identification Test (AUDIT) (Babor et al., 2001), the Structured Clinical Interview for DSM-IV Disorders (SCID) (First et al., 2002) and the Wechsler Abbreviated Scale for Intelligence (WASI) (Wechsler, 1999), respectively. Consistent with previous methods (Wesley et al., 2011; Wesley et al., 2016), individuals with current Axis I disorders (excluding alcohol use disorder) or an IQ of less than 80 were excluded from further participation.

Three experimental groups were established based on AUDIT and SCID evaluations. Individuals who scored 4 or less on the AUDIT, indicating a history of minimal alcohol consumption and no alcohol related problems, were considered a control group for heavy drinkers (CTRL; $\mathrm{n}=11$ ). Individuals who scored 8 or more indicating a history of considerable alcohol consumption and alcohol related problems were operationally defined as heavy drinkers $(n=23)$. Heavy drinkers were further divided into individuals who did and did not meet criteria for alcohol dependence based on previously established SCID criteria (Conigrave et al., 1995) and received additional questions about their alcohol use patterns. Individuals who did not meet criteria for current alcohol dependence (i.e., two or less criteria) were placed in the heavy- drinking alcohol nondependent group (A-ND; $\mathrm{n}=11$ ), whereas those who met criteria for current alcohol dependence (i.e., three or more criteria) were placed in the heavy-drinking alcohol dependent group (A-D; $n=12$ ). Worth noting is that the criteria used to define alcohol dependence under the DSM-IV overlap to a large extent with those used to define alcohol use disorder (AUD) under the current DSM-V, and likewise, the characterization of alcohol dependence and severity of AUD relates to the number of criteria met. Therefore, heavy-drinking subjects in the present study would likely have been separated into two distinct groups under the DSM-V as well (e.g., no/mild AUD vs. moderate/severe AUD). Once assigned to an experimental group, individuals were scheduled for a second laboratory visit. They were informed that during the second visit they would perform a working memory task while inside an MRI machine and were once again asked to abstain from alcohol for at least twelve hours prior to their scheduled visit.

\subsection{Procedure}

On the day of scanning, participants arrived at the laboratory approximately 2 hours prior to entering the MRI scanner. Expired air and urine samples were collected again and test for alcohol, illicit drugs and pregnancy. No participants were excluded from participation based on these screening tests. To assess anxiety levels at the time of scanning, participants completed the Spielberger State-Trait Anxiety Scale (STAI) (Spielberger et al., 1983). Participants were also asked about and monitored for signs of alcohol withdrawal. Withdrawal signs and symptoms were assessed by self-report at intake and monitored during the session by a trained laboratory technician. Participants were asked if they had experienced unusual tremors, anxiety, nausea, headaches, sweating, irritability, confusion, 
insomnia, nightmares or high blood pressure in the past twenty-four hours. No participants reported abnormal levels of any of these symptoms, and did not differ from controls on a measure of state anxiety at the time of testing (see Table 1). Next, participants completed components of the CANTAB neuropsychological battery (Cambridge Cognition, Cambridge, UK), including Delayed Match to Sample (DMS; visual working memory assessment), Intradimensional/Extradimensional Shift (ID/ED; cognitive flexibility assessment) and Stockings of Cambridge (SOC; a spatial planning assessment).

Approximately $30 \mathrm{~min}$ prior to entering the scanner, participants were familiarized with the $\mathrm{N}$-Back task on a laptop computer and the response apparatus to be used in the scanner. Task instructions were read aloud by a study technician and each participant practiced the N-Back task. Once participants were familiar with the task, they were given a 15 min break before entering the scanner. During this time, tobacco cigarette smokers were allowed to smoke one cigarette to minimize potential nicotine withdrawal effects during data collection (Wang et al., 2007; Xu et al., 2007). No members of the CTRL group, one member of the A-ND and one member of the A-D group took advantage of the opportunity to smoke a cigarette before the neuroimaging scanning session. Given the small number of individuals per group who chose to smoke a cigarette, these data were not included as a covariate in analyses. The average number of self-reported cigarettes smoked by group members, less than a standard pack of 20 cigarettes in each group (see Table 1), was included as nuisance variables in all analyses.

\subsection{N-Back task}

The N-back task used in the current study was a visual-based letter variant with three conditions (0-Back, 1-Back and 2-Back) (Callicott et al., 1999). During each condition, participants viewed a sequence of randomized white letters presented one at a time on a black background (i.e., B, G, P, T, V and X). Each time a letter appeared, participants pressed one of two buttons on a response box positioned under their right hand. For the 0 Back condition, participants were instructed to press button 1 (i.e., match response) with their index finger each time the letter " $X$ " appeared on the screen and to press button 2 (i.e., mismatch response) with their middle finger each time a different letter appeared. As such, the 0 -Back condition required attention and a behavioral response, but did not engage working memory. During the 1-Back condition, participants made a match response if the letter currently displayed on the screen was identical to letter shown 1 presentation previously (e.g., viewing sequence: B, G, T, T, G, T, G, G; correct responses: _, 2, 2, 1, 2, 2, $2,1)$. For the 2-Back condition, participants made a match response if the letter currently displayed on the screen was identical to the letter shown 2 presentations previously (e.g., viewing sequence: B, G, T, T, G, T, G, G; correct responses: _,_, 2, 2, 2, 1, 1, 2). The 1Back and 2-Back conditions therefore represent low- and high- working memory load, respectively. Figure 1A illustrates the relationship between N-Back conditions and the theoretical demand placed on neurophysiological resources during task performance.

The N-Back task was presented to the participants via MRI compatible goggles (Resonance Technology Inc., Los Angeles, CA). Each participant completed two "runs" of the N-Back task in the MRI scanner and each run contained two "blocks" of each of the three N-Back

Drug Alcohol Depend. Author manuscript; available in PMC 2018 June 01. 
task conditions (0-Back, 1-Back and 2-Back). N-Back task blocks were randomized within each run and each block started with a $5 \mathrm{~s}$ instruction screen indicating the condition about to be performed followed by a $5 \mathrm{~s}$ countdown. At the end of the countdown, a sequence of 12 letters was presented one at a time for $500 \mathrm{~ms}$ and button presses were recorded for each letter presentation. The inter-stimulus interval between each letter presentation was $2000 \mathrm{~ms}$, such that each block lasted a total of $40 \mathrm{~s}$. Each block was separated by a $12 \mathrm{~s}$ interval, so that the total task time lasted approximately $20 \mathrm{~min}$.

\section{4 fMRI data acquisition}

Images were acquired on a $1.5 \mathrm{~T}$ General Electric scanner with a birdcage-type standard quadrature head coil and an advanced nuclear magnetic resonance echoplanar system. The head was positioned along the canthomeatal line. Foam padding was used to limit head motion. Higher-resolution T1-weighted anatomical images (3D SPGR, relaxation time (TR) $=14 \mathrm{~ms}$, excitation time $(\mathrm{TE})=7700 \mathrm{~ms}$, flip angle $=25^{\circ}$, voxel dimensions $1.0 \times 1.0 \times 1.0$ $\mathrm{mm}, 176 \times 256$ voxels, 160 slices) were acquired for co-registration and normalization of functional images. A total of 200 co-planar functional images were acquired during each run of the N-Back task using a gradient echoplanar sequence $(\mathrm{TR}=2100 \mathrm{~ms}, \mathrm{TE}=40 \mathrm{~ms}$, flip angle $=90^{\circ}$, voxel dimensions $3.75 \times 3.75 \times 5.0 \mathrm{~mm}$ ). Two radio frequency excitations were performed prior to image acquisition to achieve steady-state transverse relaxation. The scanning planes were oriented parallel to the anterior commissure-posterior commissure line and extended from the superior extent of motor cortex to the base of the cerebellum. Nine volumes of data were acquired during the $20 \mathrm{~s}$ countdown period before each run and immediately discarded to allow for equilibrium before task onset.

\subsection{Statistical analyses: Demographics and Behavior}

One-way analyses of variance (ANOVAs) were used to compare the three experimental groups on AUDIT scores, age, IQ and cigarette use. Chi-square tests were used to compare groups on sex and race/ethnicity-related nonparametric variables. Independent samples ttests were used to compare A-ND and A-D groups on alcohol drinking-related variables. One-way ANCOVAs with nicotine use (cigarettes per day) as a covariate were used to compare groups on CANTAB tasks (DMS percent correct, ID/ED total errors and SOC number of problems solved in minimum moves) and N-Back reaction times.

Two approaches were used to analyze behavioral accuracy on the N-Back task. For the first approach, which was consistent with typical N-Back accuracy analyses, a percentage based accuracy score was calculated for every participant for each N-Back condition. Accuracy was calculated as: Accuracy $=[($ hits - hits possible $) /($ false alarms - false alarms possible $) \times$ 100]. A3 $\times 3$ mixed model ANCOVA, with cigarettes per day as a covariate, was performed with N- Back condition as the within group factor (i.e., 0-Back, 1-Back and 2-Back) and experimental group as the between group factor (i.e., CTRL, A-ND and A-D). When Mauchly's Test of Sphericity was significant (e.g., within group variability differed across N-Back conditions), degrees of freedom were adjusted using the Greenhouse-Geisser correction. To test for group differences within each N-Back condition, one-way ANCOVAs for independent samples (i.e., CTRL, A-ND and A-D) were conducted for each N-Back condition. Bonferonni tests were utilized for all post-hoc comparisons.

Drug Alcohol Depend. Author manuscript; available in PMC 2018 June 01. 
The second approach examined how behavioral accuracy changed in response to step- wise increases in neurophysiological demand. Accuracy change scores were calculated for 0Back to 1-Back and 1-Back to 2-Back. The difference in accuracy between 1-Back and 0Back was defined as a Low Demand transition and the difference in accuracy between 2Back and 1- Back was defined as a High Demand transition. A $2 \times 3$ mixed model ANCOVA, with cigarettes per day as a nuisance variable, was performed with demand level as the within group factor (i.e., Low Demand and High Demand) and experimental group as the between group factor (i.e., CTRL, A-ND and A-D). When Mauchly's Test of Sphericity was significant, degrees of freedom were adjusted using the Greenhouse-Geisser correction. To test for group differences at each demand level, one-way ANCOVAs for independent samples (i.e., CTRL, A-ND and A-D) were conducted at each demand level. Bonferonni tests were used for post-hoc multiple comparisons analyses. Behavioral analyses were conducted using the Statistical Package for the Social Sciences (SPSS; version 22.0) with minimum statistical significance thresholds set at $\mathrm{p}<0.05$.

\subsection{Statistical analysis: fMRI preprocessing and data analysis}

As described previously (Wesley et al., 2011), each participant's neuroimaging data were preprocessed using standard techniques. Functional volumes were corrected for slice acquisition time (i.e., slice timing correction) and realigned to the first volume acquired (i.e., motion correction). Each participant's structural volume then functional volumes were warped into standardized neuroanatomical space (Montreal Neurological Institute). Next, functional volumes were smoothed using a Gaussian $8 \mathrm{~mm}$ kernel and high-pass filtered (128s) to remove low frequency noise.

For each participant, a first-level fixed effects analysis was performed to isolate brain activity associated with each N-Back block type (i.e., 0-Back, 1-Back and 2-Back). Each block type was entered as an explanatory variable in a general linear model. Head movement parameters obtained from motion correction were entered as explanatory nuisance variables. Blood oxygen-level dependent (BOLD) signals associated with explanatory variables were convolved with a hemodynamic response function and statistical contrast maps were generated that reflected activity during each N-Back condition. These contrast maps were then carried forward into second-level within and between group random effects analyses.

Two analytical approaches were used to examine within and between group brain activity during the N-Back task. Figure 1B illustrates (1) typical and (2) step-wise analytical approaches and their relationship to N-Back conditions and the demand placed on neurophysiological resources. The typical approach focused on brain activity at low and high working memory load by comparing each load condition to the control condition (i.e., 1Back vs. 0-Back; 2-Back vs. 0- Back). The step-wise approach focused on brain activity associated with increasing neurophysiological demand. Low Demand activity was calculated by also contrasting maps of 0- Back versus 1-Back (i.e., increased activity $=1$-Back $>0$ Back; decreased activity $=1$-Back $<0$ - Back) whereas High Demand was unique in that activity was calculated by contrasting activity maps of 1-Back versus 2-Back (i.e., increased activity $=2$-Back $>1$-Back; decreased activity $=2$-Back $<1$-Back). Note that the analysis of brain activity during the low working memory load condition is identical to the step-wise

Drug Alcohol Depend. Author manuscript; available in PMC 2018 June 01. 
Low Demand transition (i.e., 0-Back vs. 1-Back), so the results from this analysis are only presented once.

Within group analyses utilized one-sample t-tests with voxel-wise probability thresholds set to $\mathrm{p}<0.001$ and spatial cluster extents set to 25 contiguous voxels. Between group analyses utilized independent samples t-tests with voxel-wise probability thresholds set to $\mathrm{p}<0.01$ and spatial cluster extents set to 25 contiguous voxels. Multiple comparison correction was performed using small volume family-wise error correction ( $F E W, \mathrm{p}<0.05)$. Number of cigarettes per day was included as a variable of no interest in all second-level models. Data preprocessing and analyses were performed with custom MATLAB 7.0 (Mathworks, Natick, MA) scripts and standard statistical parametric mapping (SPM5; Wellcome Department of Cognitive Neurology, London, UK; (Ashburner and Friston, 2005)).

\subsection{Results}

Grouping, demographic, alcohol- and other cognition-related variables are shown in Table 1.

\subsection{Group Assignment}

Consistent with group assignments the AUDIT score was significantly greater in A-D, compared A-ND and CTRL. Additionally, A-D had significantly greater alcohol dependence symptoms, compared to A-ND, but these groups did not differ in other drinking-related variables, including age of first drink, drinks per week, length of current drinking pattern and time since last drink.

\subsection{Demographics and Other Variables}

Groups did not differ in age, IQ, sex or race/ethnicity. Groups also did not differ in state or trait anxiety at the time of data collection. A-D smoked significantly more cigarettes per day than CTRL; therefore, the number of cigarettes smoked per day was added as a nuisance variable in behavioral and neuroimaging analyses. CANTAB task performance and N-Back reaction times are also reported in Table 1. CANTAB performance did not differ between groups on the ID/ED task (a measure of cognitive flexibility) or the SOC task (a measure of spatial planning). Groups did differ, however, on the DMS task (a measure of visual working memory; $F 2,32=4.5, \mathrm{p}<0.02$ ), with a significantly lower percentage of correct responses in A-D compared to A-ND. Groups did not differ in N-Back reaction times.

\subsection{N-Back Performance Accuracy}

Accuracy scores for each N-Back condition are shown in Figure 2A. A significant condition by group interaction was observed $(\mathrm{F} 2,32=3.35, p<0.05)$. Follow-up tests revealed a significant group effect for the 0 -Back condition $(\mathrm{F} 2,35=3.57, p<0.04)$, with accuracy in A$\mathrm{D}(0.86 \pm 0.03$; mean \pm S.E. $)$ being significantly less than that of CTRL $(0.94 \pm 0.01 ; p<0.04)$ but not A-ND (0.91 \pm 0.02$)$. Accuracy did not differ between groups during the 1-Back condition (F2,35=1.99, $p<0.15$ ), with similar values observed for CTRL (0.88 \pm 0.02$)$, A-ND $(0.93 \pm 0.01)$ and A-D $(0.87 \pm 0.02)$. During the 2-Back condition, however, a group effect was observed ( $\mathrm{F} 2,35=5.7, p<0.008)$, with accuracy in the A-D (0.58 .06) group being significantly less than both CTRL $(0.78 \pm .04 ; p<0.04)$ and A-ND $(0.77 \pm .06)$. 


\subsection{Brain Activity During High Working Memory Load}

A typical analysis of brain activity during the low working memory load condition (0- Back vs. 1-Back) is identical to the step-wise Low Demand transition, so the results from this analysis are only presented once, in a subsequent section. Typical analysis of within group brain activity during the high working memory load condition of the N-Back task (0-Back vs. 2-Back) revealed similar general patterns of increased (2-Back $>0$-Back) and decreased (2-Back < 0- Back) activity in all groups (Figure S1). Each group displayed increased activity in bilateral dorsal lateral prefrontal cortex (DLPFC), superior parietal cortex (sPar) and dorsal medial prefrontal cortex (dmPFC) during 2-Back, compared to 0-Back.

Decreased activity was observed in the ventral medial prefrontal cortex (vmPFC) in A-D and A-ND, and all groups showed decreased activity in the posterior cingulate cortex (PCC) during 2-Back, compared 0-Back. Between groups comparisons revealed that AD had significantly greater activity in bilateral DLPFC and vmPFC, compared to CTRL, during 2Back relative to 0-Back (Figure 2B).

\subsection{Accuracy and Brain Activity Associated with Transition to Low Demand}

Behavior and brain activity associated with the transition to Low Demand (0-Back to 1Back) is shown in Figure 3. Groups did not differ in accuracy change scores associated with the transition to Low Demand (Figure 3A; F2, 35=2.6, $p<0.09)$. The mean $( \pm$ S.E.) differences in percentage accuracy scores between 0-Back and 1-Back in CTRL, A-ND and A-D were - 0.06(.02), 0.02(.02) and 0.01(.03), respectively. Within group analyses of the transition to Low Demand, however, revealed some general similarities and differences in activity patterns within the groups (Figure 3B; Table 2A). CTRL displayed decreased activity in the posterior cingulate cortex (PCC; cluster size: $k \mathrm{E}=248)$ and increased activity in the superior parietal cortex (sPar). By comparison, similar activity patterns were observed in heavy drinkers but were larger in number, magnitude and spatial extent. For example, decreased activity in the PCC of A-ND and A-D occurred in clusters sized $k E=5155$ and $k \mathrm{E}=4588$, respectively. Decreased activity was also observed in the medial prefrontal cortex (mPFC) within A-ND and A-D, but not CTRL. Lastly, increased activity was observed in bilateral DLPFC within A-ND and A-D, but not CTRL. Suprathreshold clusters did not survive between group testing criteria (Table 2B).

\subsection{Accuracy and Brain Activity Associated with Transition to High Demand}

Behavior and brain activity associated with the transition from Low Demand to High Demand (1-Back to 2-Back) is shown in Figure 4. Groups significantly differed in accuracy change scores associated with the transition from Low Demand to High Demand (Figure $4 \mathrm{~A} ; \mathrm{F} 2,35=4.59, p<0.02)$. Post hoc analysis revealed that the percentage accuracy difference between 1-Back and 2-Back was significantly decreased in A-D (-0.28土.05), relative to CTRL $(-0.10 \pm .03)$. The percentage accuracy difference in A-ND $(-0.16 \pm .06)$ fell between that of CTRL and A-D and did not significantly differ from either group.

Within group brain activity associated with the transition from Low Demand to High Demand is displayed in Figure 4B and Table 3A. Increased activity was observed in CTRL in the dorsal medial prefrontal cortex (dmPFC), precuneus (prCun), bilateral sPar and the right DLPFC. Decreased activity was observed in the ventral medial prefrontal cortex 
(vmPFC) of CTRL. Note that the mPFC effects observed in CTRL are more ventral than those observed in A-D during the transition to Low Demand (0-Back vs. 1-Back). A-ND did not show additional decreased activity during the transition to High Demand, but displayed large clusters of increased activity in the dmPFC, prCun, bilateral sPar and bilateral DLPFC. A-D displayed minimal increased activity in bilateral sPar, dmPFC and right DLPFC.

Analysis of between-group differences in brain activity associated with the transition from Low Demand to High Demand revealed significant differences between CTRL and A-D as well as A-ND and A-D, but not CTRL and A-ND (Figure 5; Table 3B). Specifically, the increased activity in the left sPar of A-ND was significantly greater than sPAR activity observed in A-D. Additionally, there was a significantly larger decrease in mPFC activity observed in CTRL compared to A-D, indicating that the greater mPFC activity observed in A-D compared to CTRL from the typical 0-Back versus 2-Back analysis was at least partially driven by the significant hypoactive responses in ventral portions of mPFC in CTRL associated with the transition from 1-Back to 2-Back.

\subsection{Discussion}

Reduced working memory performance, as assessed by the N-Back task, has been demonstrated in individuals diagnosed with a range of mental health conditions, including alcohol dependence (Pitel et al., 2007; Pitel et al., 2009). Consistent with those prior results, the present study revealed that individuals in the alcohol-dependent (A-D) group were less accurate on the 2-Back condition of the task than participants in the nondependent heavy drinker (A-ND) and control (CTRL) groups.

The majority of the neurophysiological changes (i.e., energy expenditure as reflected by oxygen utilization and detected with fMRI) observed in the current study are consistent with previous findings from studies that used neuroimaging techniques to determine the neural correlates of working memory. Typical within-group analyses comparing brain activity during the zero and high load conditions of the task (0-Back vs. 2-Back; see supplementary data ${ }^{1}$ ) revealed that all three groups independently utilized brain regions previously demonstrated to be involved in attention and working memory processes, including the bilateral dorsal lateral prefrontal cortex (DLPFC), superior parietal cortex (sPar) and dorsal medial prefrontal cortex (dmPFC). All groups also independently displayed decreased activity in default-mode network brain areas, including portions of the medial prefrontal cortex (mPFC) and posterior cingulate cortex (PCC). That imposing task demands results in decreased activity in default-mode related brain areas and increased activity in attention and cognitive control brain areas is a finding consistent across neuroimaging studies using a variety of tasks to measure cognitive performance. Group comparisons of brain activity during the high working memory load condition (0-Back vs. 2-Back) revealed that A-D subjects had greater activity in both the MPFC and bilateral DLPFC compared to CTRL. Thus, A-D subjects displayed greater activity in portions of both default and attention/ cognitive control networks during high working memory demand, suggesting that their impaired task performance might be related to an inability to suppress mPFC activity despite

\footnotetext{
${ }^{1}$ Supplementary material can be found by accessing the online version of this paper at http://dx.doi.org and by entering doi:...
} 
compensatory increases in the DLPFC to meet greater task demand. However, a limitation of that analytic approach and subsequent interpretation is that the comparison of 0-Back to 2Back also includes physiological changes attributable to the low demand condition of the task (1-Back). Therefore, to better understand the relationship between the increasing demand placed neurophysiological resources and its relationship to problematic drinking, we initiated a step-wise analysis examining changes in behavior and brain activity associated with transitioning to a Low Demand state (e.g., 0-Back to 1-Back) and further to a High Demand state (e.g., 1-Back to 2-Back).

The transition to Low Demand did not differentially impact task performance across groups, whereas the transition to High Demand resulted in impaired accuracy in A-D relative to CTRL. In general, the results from the within-group analyses were suggestive of greater overall functional engagement at earlier demand in heavy drinkers relative to controls (compare within- group images in Fig. 2B to Fig. 3B). However, between-group comparisons only revealed significant differences in regional brain activity associated with the transition to the High Demand, which parallels the behavioral results. The results from those analyses indicated that A- ND had significantly greater activity in the sPar compared to A-D, and that mPFC activity was decreased to a greater degree in CTRL relative to A-D, during the transition to High Demand. The sPar is part of a dorsal processing stream that is related to attention and associated with performance on experimental tasks measuring various cognitive domains, including attention and memory (Kumfor et al., 2015; Rosen et al., 2015). For example, the sPar was found to mediate visuospatial attention in a recent transcranial magnetic stimulation study (Wu et al., 2016). The mPFC, on the other hand, is part of the default-mode network and decreased activity in that network is consistent with a shift to a more attention-focused brain state (Raichle and Snyder, 2007; Wang and Li, 2013). As noted above, a common interpretation is that activity in default- mode networks must decrease (Koshino et al., 2014) and/or desynchronize (Gao et al., 2013) as neurophysiological resources are reallocated to meet experimental task demands. Inability to deactivate or disengage the $\mathrm{MPFC}$ has been associated with lapses in attention (Weissman et al., 2006) and increased errors on executive functioning tasks (Li et al., 2007), and damage to the mPFC was shown to impair performance on the N-Back task (Tsuchida and Fellows, 2009). Together, these data suggest that limited engagement of the sPar and an inability to fully disengage the mPFC might be responsible for the impaired task performance observed in A-D at High Demand. Further, these between-group mPFC findings during the transition to High Demand are in agreement with the more typical analysis results reported above and suggest that the greater $\mathrm{mPFC}$ responses in A-D vs. CTRL during high working memory load (0-Back vs. 2- Back) were driven at least in part by decreasing mPFC activity in CTRL associated with the transition from low (1-Back) to high (2-Back) demand (confirmed by the within-group results).

One limitation of the present study is that more detailed information about participants' drinking patterns, in particular the number of drinks consumed on the same occasion, was not collected at the time the study was conducted. Participants were assigned to the control versus heavy drinking groups according to AUDIT scores, with individuals who scored 4 or less on the AUDIT being considered controls and individuals who scored 8 or greater considered heavy drinkers. However, the current categorization of heavy drinking by the 
United States National Institute of Alcohol Abuse and Alcoholism and the Substance Abuse and Mental Services Health Administration (SAMHSA) relies on information about drinks consumed in a single occasion. More specifically, heavy drinking is defined as consuming 5 or more drinks on the same occasion on each of 5 or more days in the past 30 days (SAMHSA, 2014). Notwithstanding, the World Health Organization has used the AUDIT as an assessment tool for defining alcohol consumption and therefore the current results may more readily translate to international assessments of alcohol consumption. Future studies will include updated SAMHSA criteria when establishing experimental groups and will also focus on how the observed effects may relate to long-term alcohol dependent heavy drinkers (e.g., > 20 years) as well as individuals who do not yet report heavy consumption, but who are at-risk for alcohol abuse due to positive family history of alcoholism (FH+). For example, evidence if $\mathrm{FH}+$ subjects showed the same decreased activation patterns to the High Demand transition compared with FH- subjects, those results would suggest that the differential behavior and brain profile observed here is a precursor to heavy alcohol consumption rather than a consequence. Lastly, because the current study did not record brain activity during a resting state, group differences in default-mode network activity during the transition into task engagement (i.e., 0-Back) could not be determined. Future studies should include the acquisition of resting state data in the same scanning session in order to model a larger range of neurophysiological transitions.

\subsection{Conclusion}

In conclusion, the behavioral and brain changes observed in the current study suggest that working memory deficits in alcohol dependent individuals are associated with abnormal function of default and cognitive control brain networks in response to increasing task demands. These results further suggest that alcohol dependent individuals have less capacity for meeting the demand of increasing working memory loads. From a treatment standpoint, these results highlight two brain areas that could be targeted to improve clinical outcomes; namely, the mPFC (decreased response differentiating CTRL and A-D) and left sPar (increased response observed in A-ND but not A-D). One strategy is to enhance function in these areas through noninvasive brain stimulation or cognitive training combined with tasks known to engage them. For example, working memory training has been used to shift temporal discounting rates in cocaine users (Bickel et al., 2011) and it has been hypothesized that this training increases functional capacity in cognitive control networks (Wesley and Bickel, 2014). This approach might also be beneficial in alcohol dependent individuals, who have been shown to devalue future rewards (Bobova et al., 2009; Mitchell et al., 2005; Petry, 2001). Functional enhancement of these target brain areas, resulting in the ability to meet larger external demand, may lead to more effective treatment outcomes in alcohol use disorder.

\section{Supplementary Material}

Refer to Web version on PubMed Central for supplementary material. 


\section{Acknowledgments}

The authors would like to thank Dr. Erin E. Shannon for her contribution to data collection and analysis, Marla C. Torrence for her involvement in subject screening and data collection, and Mack D. Miller for his assistance with task design. The authors would also like to thank Jenna C. Przybylowski for her beneficial advice, laboratory leadership, engagement in research and assistance with manuscript preparation.

Funding: This work was supported by National Institute of Drug Abuse [grant numbers DA007246, DA031766, DA020074, and DA06634].

\section{References}

Albein-Urios N, Martinez-Gonzalez JM, Lozano O, Clark L, Verdejo-Garcia A. Comparison of impulsivity and working memory in cocaine addiction and pathological gambling: Implications for cocaine-induced neurotoxicity. Drug Alcohol Depend. 2012; 126:1-6. [PubMed: 22475814]

Ashburner J, Friston KJ. Unified segmentation. Neuroimage. 2005; 26:839-851. [PubMed: 15955494]

Babor, TF., Higgins-Biddle, JC., Saunders, JB., Monteiro, MG. The Alcohol-Use Disorders Identification Test (Audit) — Guidlines for Use in Primary Care. World Health Organization; 2001.

Barkley RA. Behavioral inhibition, sustained attention, and executive functions: Constructing a unifying theory of ADHD. Psychol Bull. 1997; 121:65-94. [PubMed: 9000892]

Bickel WK, Yi R, Landes RD, Hill PF, Baxter C. Remember the future: Working memory training decreases delay discounting among stimulant addicts. Biol Psychiatr. 2011; 69:260-265.

Bobova L, Finn PR, Rickert ME, Lucas J. Disinhibitory psychopathology and delay discounting in alcohol dependence: personality and cognitive correlates. Exp Clin Psychopharmacol. 2009; 17:5161. [PubMed: 19186934]

Callicott JH, Mattay VS, Bertolino A, Finn K, Coppola R, Frank JA, Goldberg TE, Weinberger DR. Physiological characteristics of capacity constraints in working memory as revealed by functional MRI. Cereb Cortex. 1999; 9:20-26. [PubMed: 10022492]

Charlet K, Beck A, Jorde A, Wimmer L, Vollstadt-Klein S, Gallinat J, Walter H, Kiefer F, Heinz A. Increased neural activity during high working memory load predicts low relapse risk in alcohol dependence. Addict Biol. 2014; 19:402-414. [PubMed: 24147643]

Conigrave KM, Hall WD, Saunders JB. The AUDIT questionnaire: Choosing a cut-off score. Alcohol Use Disorder Identification Test. Addiction. 1995; 90:1349-1356. [PubMed: 8616463]

Cremaschi L, Penzo B, Palazzo M, Dobrea C, Cristoffanini M, Dell'Osso B, Altamura AC. Assessing working memory via N-back task in euthymic bipolar I disorder patients: a review of functional magnetic resonance imaging studies. Neuropsychobiology. 2013; 68:63-70. [PubMed: 23881005]

Duarte NA, Woods SP, Rooney A, Atkinson JH, Grant I, Translational Methamphetamine A.R.C.G. Working memory deficits affect risky decision-making in methamphetamine users with attentiondeficit/hyperactivity disorder. J Psychiatr Res. 2012; 46:492-499. [PubMed: 22305489]

Elzinga BM, Ardon AM, Heijnis MK, De Ruiter MB, Van Dyck R, Veltman DJ. Neural correlates of enhanced working-memory performance in dissociative disorder: A functional MRI study. Psycholog Med. 2007; 37:235-245.

Fillmore MT. Drug abuse as a problem of impaired control: Current approaches and findings. Behav Cogn Neurosci Rev. 2003; 2:179-197. [PubMed: 15006292]

Fillmore MT, Van Selst M. Constraints on information processing under alcohol in the context of response execution and response suppression. Exp Clin Psychopharmacol. 2002; 10:417-424. [PubMed: 12498339]

Finn PR. Motivation, working memory, and decision making: a cognitive-motivational theory of personality vulnerability to alcoholism. Behav Cogn Neurosci Rev. 2002; 1:183-205. [PubMed: 17715592]

First, MB., Spitzer, RL., Gibbon, M., Williams, JBW. Structured clinical interview for DSM-IV-TR Axis I disorders-patient edition. Biometrics Research Department; New York State Psychiatric Institute; New York, New York: 2002.

Friston K. The free-energy principle: a rough guide to the brain? Trends Cogn Sci. 2009; 13:293-301. [PubMed: 19559644] 
Friston K. The free-energy principle: A unified brain theory? Nature Rev Neurosci. 2010; 11:127-138. [PubMed: 20068583]

Friston K, Kilner J, Harrison L. A free energy principle for the brain. J Physiol Paris. 2006; 100:70-87. [PubMed: 17097864]

Gao W, Gilmore JH, Alcauter S, Lin W. The dynamic reorganization of the default-mode network during a visual classification task. Front Syst Neurosci. 2013; 7:34. [PubMed: 23898240]

Gunn RL, Finn PR. Impulsivity partially mediates the association between reduced working memory capacity and alcohol problems. Alcohol. 2013; 47:3-8. [PubMed: 23200800]

Hinson JM, Jameson TL, Whitney P. Impulsive decision making and working memory. J Exp Psychol Learn Mem Cogn. 2003; 29:298-306. [PubMed: 12696817]

Hofmann W, Gschwendner T, Friese M, Wiers RW, Schmitt M. Working memory capacity and selfregulatory behavior: Toward an individual differences perspective on behavior determination by automatic versus controlled processes. J Pers Soc Psychol. 2008; 95:962-977. [PubMed: 18808271]

Jansma JM, Ramsey NF, van der Wee NJ, Kahn RS. Working memory capacity in schizophrenia: A parametric fMRI study. Schizophr Res. 2004; 68:159-171. [PubMed: 15099600]

Koshino H, Minamoto T, Yaoi K, Osaka M, Osaka N. Coactivation of the Default Mode Network regions and Working Memory Network regions during task preparation. Sci Rep. 2014; 4:5954. [PubMed: 25092432]

Kumfor F, Dermody N, Irish M. Considering the impact of large-scale network interactions on cognitive control. J Neurosci. 2015; 35:1-3. [PubMed: 25568098]

Li CS, Yan P, Bergquist KL, Sinha R. Greater activation of the "default" brain regions predicts stop signal errors. Neuroimage. 2007; 38:640-648. [PubMed: 17884586]

Mitchell JM, Fields HL, D’Esposito M, Boettiger CA. Impulsive responding in alcoholics. Alcohol Clin Exp Res. 2005; 29:2158-2169. [PubMed: 16385186]

Niendam TA, Laird AR, Ray KL, Dean YM, Glahn DC, Carter CS. Meta-analytic evidence for a superordinate cognitive control network subserving diverse executive functions. Cogn Affect Behav Neurosci. 2012; 12:241-268. [PubMed: 22282036]

Park MS, Sohn S, Park JE, Kim SH, Yu IK, Sohn JH. Brain functions associated with verbal working memory tasks among young males with alcohol use disorders. Scand J Psychol. 2011; 52:1-7. [PubMed: 21054421]

Petry NM. Delay discounting of money and alcohol in actively using alcoholics, currently abstinent alcoholics, and controls. Psychopharmacol. 2001; 154:243-250.

Pfefferbaum A, Desmond JE, Galloway C, Menon V, Glover GH, Sullivan EV. Reorganization of frontal systems used by alcoholics for spatial working memory: An fMRI study. Neuroimage. 2001; 14:7-20. [PubMed: 11525339]

Pitel AL, Beaunieux H, Witkowski T, Vabret F, Guillery-Girard B, Quinette P, Desgranges B, Eustache F. Genuine episodic memory deficits and executive dysfunctions in alcoholic subjects early in abstinence. Alcohol Clin Exp Res. 2007; 31:1169-1178. [PubMed: 17511749]

Pitel AL, Rivier J, Beaunieux H, Vabret F, Desgranges B, Eustache F. Changes in the episodic memory and executive functions of abstinent and relapsed alcoholics over a 6-month period. Alcohol Clin Exp Res. 2009; 33:490-498. [PubMed: 19120052]

Raichle ME, Snyder AZ. A default mode of brain function: A brief history of an evolving idea. Neuroimage. 2007; 37:1083-1090. discussion 1097-1089. [PubMed: 17719799]

Rosen ML, Stern CE, Michalka SW, Devaney KJ, Somers DC. Cognitive Control Network Contributions to Memory-Guided Visual Attention. Cereb Cortex. 2015

SAMHSA. Substance Abuse and Mental Health Services Administration, Results from the 2013 National Survey on Drug Use and Health: Summary of National Findings, NSDUH Series H-48. Substance Abuse and Mental Health Services Administration; Rockville, MD: 2014. HHS Publication No. (SMA) 14-4863

Spielberger, CD., Gorsuch, RL., Lushene, R., Vagg, PR., Jacobs, GA. Manual for the State-Trait Anxiety Inventory. Consulting Psychologists Pressv; Palo Alto, CA: 1983. 
Tapert SF, Brown GG, Kindermann SS, Cheung EH, Frank LR, Brown SA. fMRI measurement of brain dysfunction in alcohol-dependent young women. Alcohol Clin Exp Res. 2001; 25:236-245. [PubMed: 11236838]

Tsuchida A, Fellows LK. Lesion evidence that two distinct regions within prefrontal cortex are critical for n-back performance in humans. J Cogn Neurosci. 2009; 21:2263-2275. [PubMed: 19199405]

Vo HT, Schacht R, Mintzer M, Fishman M. Working memory impairment in cannabis- and opioiddependent adolescents. Subst Abus. 2014; 35:387-390. [PubMed: 25148203]

Wang Y, Li TQ. Analysis of whole-brain resting-state FMRI data using hierarchical clustering approach. PloS one. 2013; 8:e76315. [PubMed: 24204612]

Wang Z, Faith M, Patterson F, Tang K, Kerrin K, Wileyto EP, Detre JA, Lerman C. Neural substrates of abstinence-induced cigarette cravings in chronic smokers. J Neurosci. 2007; 27:14035-14040. [PubMed: 18094242]

Weber EU, Johnson EJ. Mindful judgment and decision making. Annu Rev Psychol. 2009; 60:53-85. [PubMed: 18798706]

Wechsler, D. Wechsler Abbreviated Scale of Intelligence. The Psychological Corporation: Harcourt Brace and Company; New York, NY: 1999.

Weissman DH, Roberts KC, Visscher KM, Woldorff MG. The neural bases of momentary lapses in attention. Nature Neurosci. 2006; 9:971-978. [PubMed: 16767087]

Wesley MJ, Bickel WK. Remember the future II: Meta-analyses and functional overlap of working memory and delay discounting. Biol Psychiatr. 2014; 75:435-448.

Wesley MJ, Hanlon CA, Porrino LJ. Poor decision-making by chronic marijuana users is associated with decreased functional responsiveness to negative consequences. Psychiatr Res. 2011; 191:5159.

Wesley MJ, Lile JA, Hanlon CA, Porrino LJ. Abnormal medial prefrontal cortex activity in heavy cannabis users during conscious emotional evaluation. Psychopharmacol. 2016; 233:1035-1044.

Wesley MJ, Lohrenz T, Koffarnus MN, McClure SM, De La Garza R 2nd, Salas R, Thompson-Lake DG, Newton TF, Bickel WK, Montague PR. Choosing money over drugs: The neural underpinnings of difficult choice in chronic cocaine users. J Addict. 2014; 2014:189853. [PubMed: 25197609]

Wilcox CE, Dekonenko CJ, Mayer AR, Bogenschutz MP, Turner JA. Cognitive control in alcohol use disorder: Deficits and clinical relevance. Rev Neurosci. 2014; 25:1-24. [PubMed: 24361772]

Wu Y, Wang J, Zhang Y, Zheng D, Zhang J, Rong M, Wu H, Wang Y, Zhou K, Jiang T. The neuroanatomical basis for posterior superior parietal lobule control lateralization of visuospatial attention. Front Neuroanat. 2016; 10:32. [PubMed: 27047351]

Xu J, Mendrek A, Cohen MS, Monterosso J, Simon S, Jarvik M, Olmstead R, Brody AL, Ernst M, London ED. Effect of cigarette smoking on prefrontal cortical function in nondeprived smokers performing the Stroop Task. Neuropsychopharmacol. 2007; 32:1421-1428.

Drug Alcohol Depend. Author manuscript; available in PMC 2018 June 01. 


\section{Highlights}

- $\quad$ Performance fails in alcohol dependent heavy drinkers at high working memory loads

- A novel approach examined neurobehavioral changes from low to high demand states

- Heavy drinkers engaged neurofunctional resources at lower demand than controls

- Dependent heavy drinkers had diminished neural capacity at higher demand 
A.

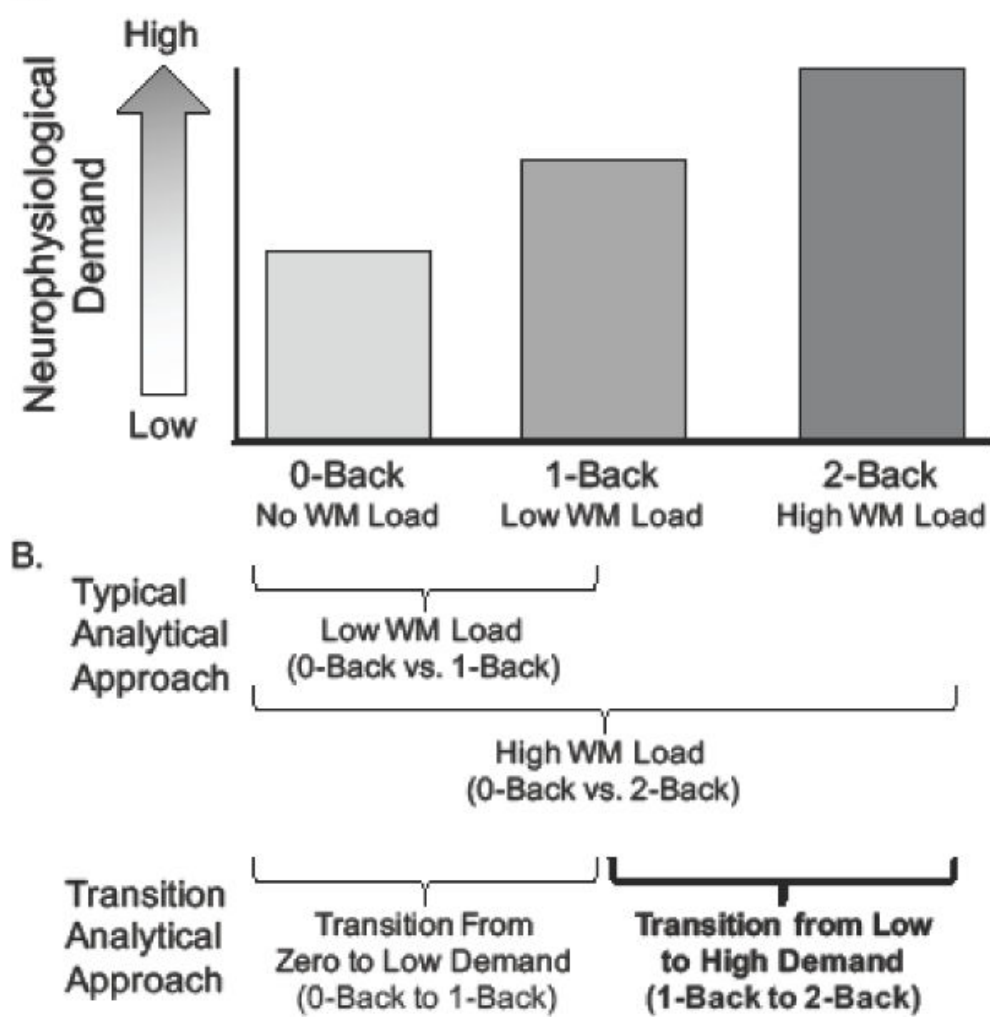

Figure 1.

The N-Back task, neurophysiological demand and different analytical approaches. (A) The three N-Back task conditions (0-, 1- and 2-Back) and their relationship to working memory (WM) load and the demand placed on neurophysiological resources. Demand is presented on the $y$-axis and task conditions are presented on the $\mathrm{x}$-axis. During the 0 -Back condition, neurophysiological demand is minimal as attention and match/mismatch responses are required but there is no WM load. During the 1-Back condition demand is increased to a low level as a low WM load is added to the task. During the 2-Back condition, demand is increased further to high level as a high WM load is added to the task. (B) A typical analytical approach isolates brain activity associated with high WM load by subtracting activity observed in the 0 -Back condition from that observed in the 2-Back condition. This approach therefore includes neurophysiological signals also required for the low WM load condition. A transitional analytical approach examines step-wise increases in demand by isolating neurophysiological signals associated with the transition between increasing $\mathrm{N}$ Back conditions $(0-$ Back to 1 -Back $=$ transition to Low Demand; 1 -Back to 2-Back $=$ transition to High Demand: bolded and unique to the current study). 


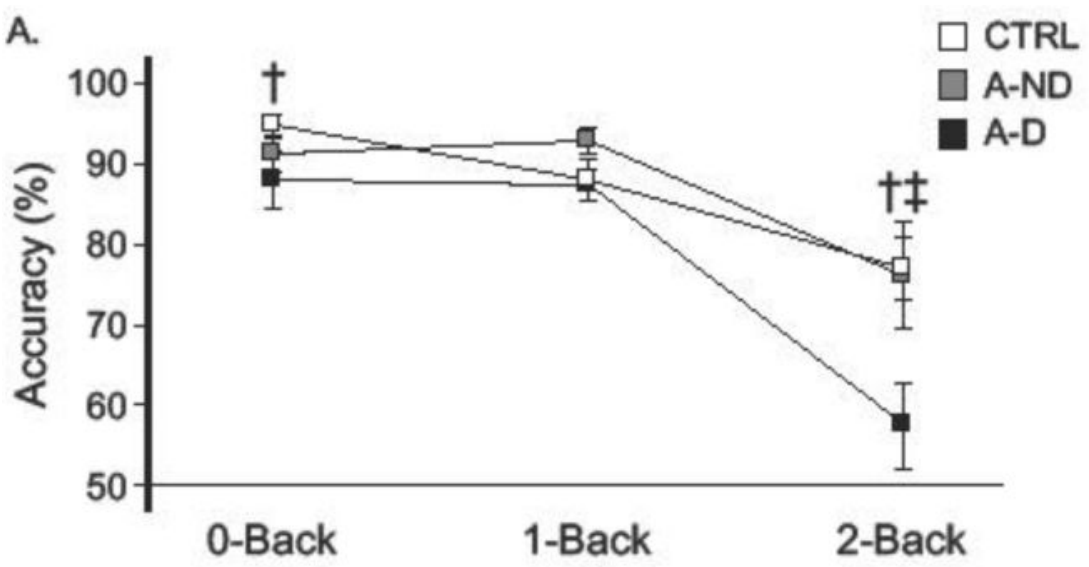

High WM Load

B. (0-Back vs. 2-Back)

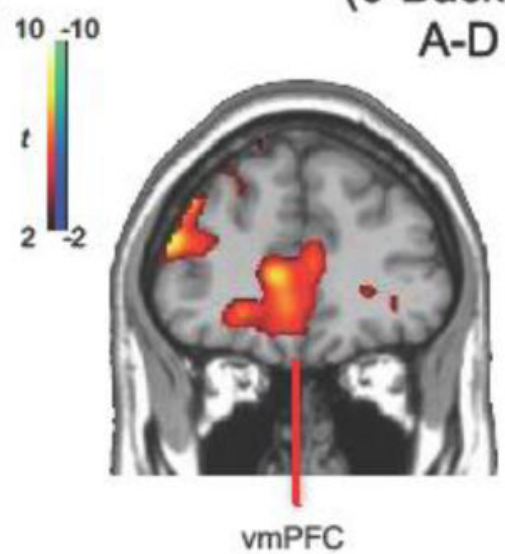

DLPFC

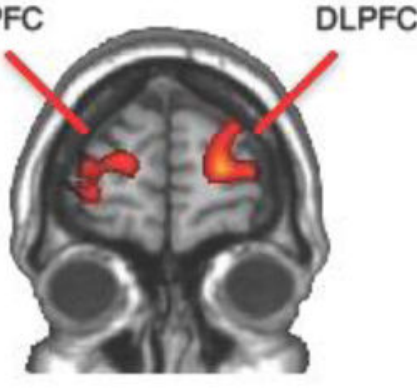

Figure 2.

N-Back performance accuracy and neuroimaging results from a typical analysis of high load working memory on the N-Back task. (A) Performance accuracy (mean \pm SEM; y- axis) across increasing N-Back conditions (0-Back, 1-Back and 2-Back; $\mathrm{x}$-axis) in controls (CTRL), heavy drinkers who were alcohol nondependent (A-ND) and heavy drinkers who were alcohol dependent (A-D). Increased working memory load was associated with reduced accuracy in each group. At the highest working memory load (2-Back), accuracy in A-D was significantly less than CTRL and A-ND ( $†$ CTRL > A-D; $\neq$ A-ND > A-D; p<0.05). (B) A typical analysis of activity at high working memory load (0-Back vs. 2-Back) revealed greater activity in cognitive control and attention brain areas, including bilateral dorsal lateral prefrontal cortex (DLPFC) and medial prefrontal cortex (mPFC), in A-D relative to CTRL. 


\section{Transition From Zero to Low Demand (0-Back to 1-Back)}

A.

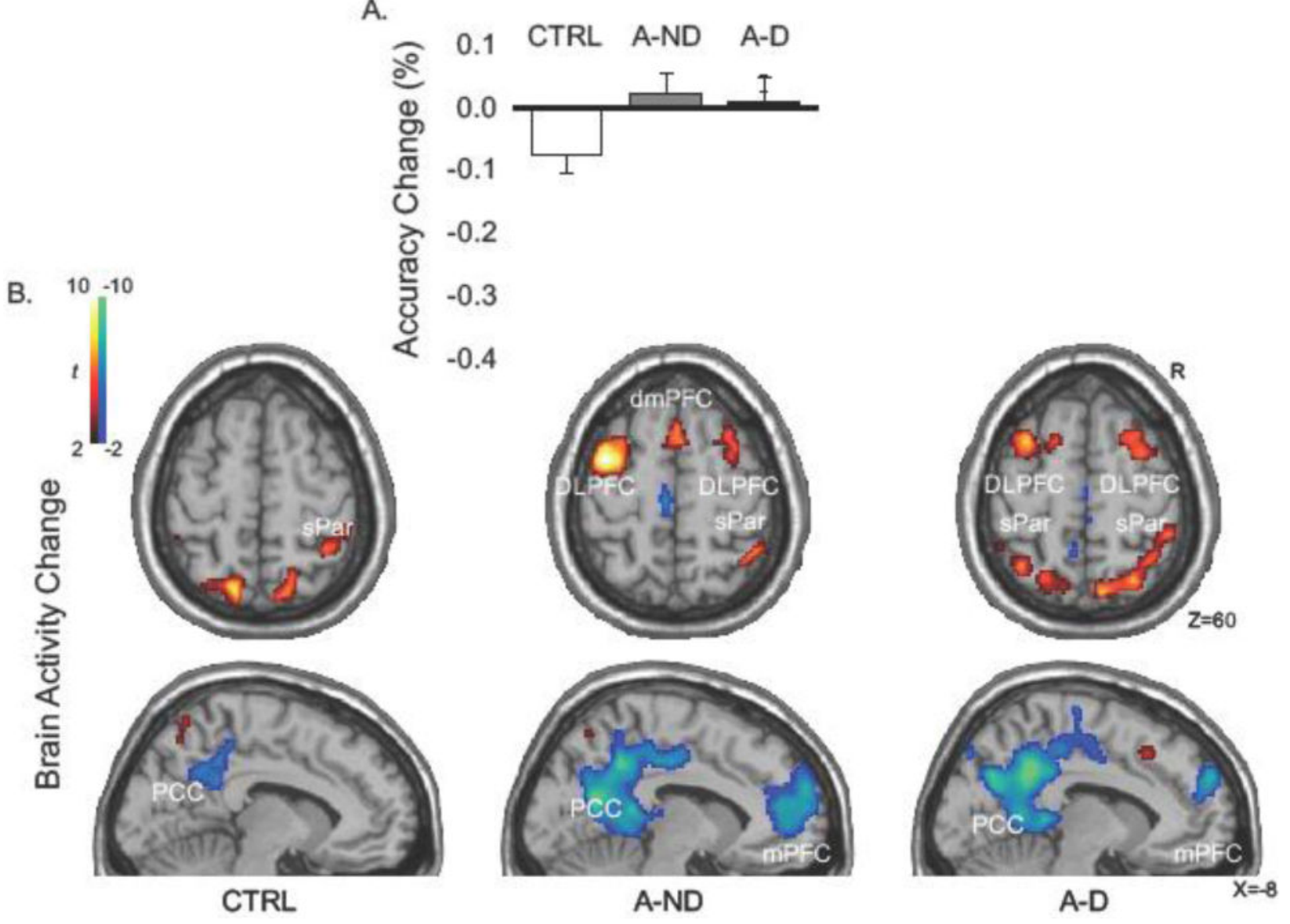

Figure 3.

Within group changes in accuracy and brain function associated with transitioning from the 0 -Back to the 1-Back conditions of the N-Back task (Low Demand) in controls (CTRL; left), heavy drinkers who were alcohol nondependent (A-ND; middle) and heavy drinkers who were alcohol dependent (A-D; right). (A) Accuracy change scores for the Low Demand transition did not vary by group. (B) Increases (1-Back > 0-Back; hot) and decreases (1Back < 0-Back; cold) in brain activity associated with the transition to the Low Demand state. Voxel- level probability thresholds were set to $\mathrm{p}<0.001$ and corrected at the cluster level $(F W E, \mathrm{p}<0.05) . \mathrm{R}=$ right hemisphere. $\mathrm{sPar}=$ superior parietal cortex. $\mathrm{PCC}=$ posterior cingulate cortex. $\mathrm{dmPFC}=$ dorsal medial prefrontal cortex. $\mathrm{DLPFC}=$ dorsal lateral prefrontal cortex. $\mathrm{mPFC}=$ medial prefrontal cortex. 


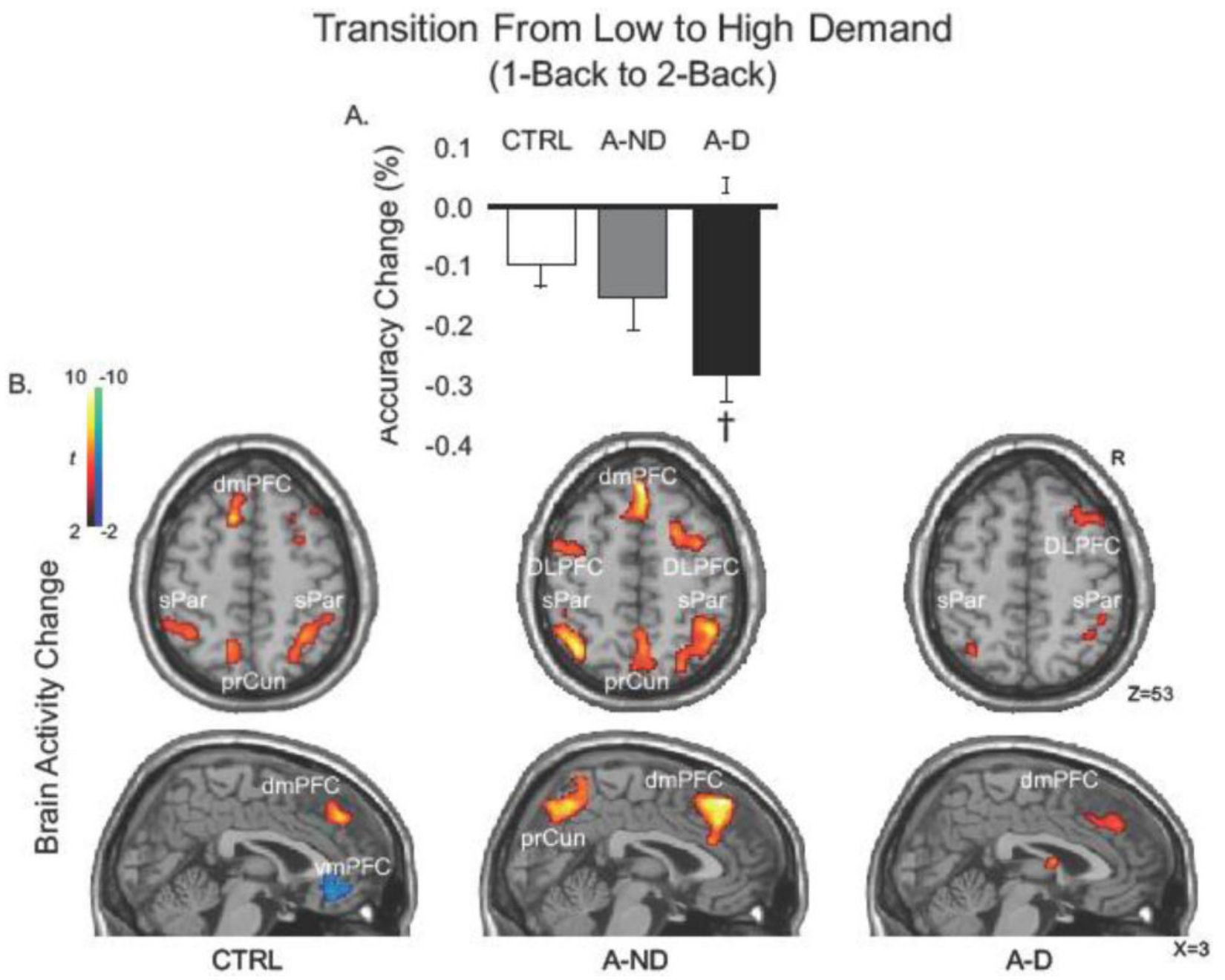

Figure 4.

Within group changes in accuracy and brain function associated with transitioning from the 1-Back to the 2-Back (High Demand) conditions of the N-Back task in controls (CTRL; left), heavy drinkers who were alcohol nondependent (A-ND; middle) and heavy drinkers who were alcohol dependent (A-D; right). (A) Accuracy change scores of A-D were significantly reduced relative to CTRL during the transition to the High Demand state. (B) Increases (2-Back > 0-Back; hot) and decreases (2-Back < 0-Back; cold) in brain activity associated with the transition to the High Demand state. Voxel-level probability thresholds were set to $\mathrm{p}<0.001$ and corrected at the cluster level $(F W E, \mathrm{p}<0.05) . \mathrm{R}=$ right hemisphere. $\mathrm{dmPFC}=$ dorsal medial prefrontal cortex. DLPFC $=$ dorsal lateral prefrontal cortex $. \mathrm{sPar}=$ superior parietal cortex prCun $=$ Precuneus. $\mathrm{PCC}=$ posterior cingulate cortex. $\mathrm{vmPFC}=$ ventral medial prefrontal cortex. 


\section{Transition From Low to High Demand} (1-Back to 2-Back)

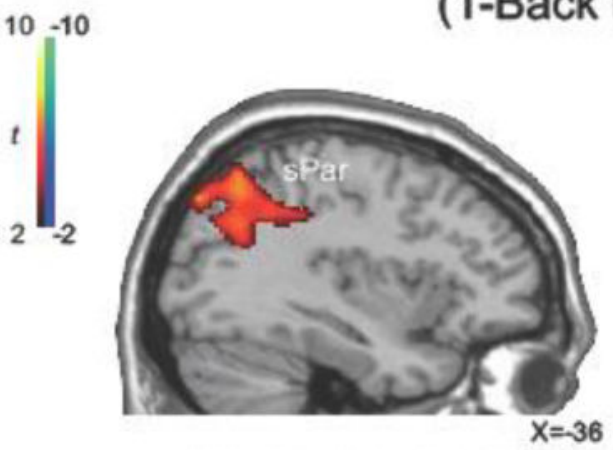

Increased in A-ND compared to A-D (A-ND > A-D)

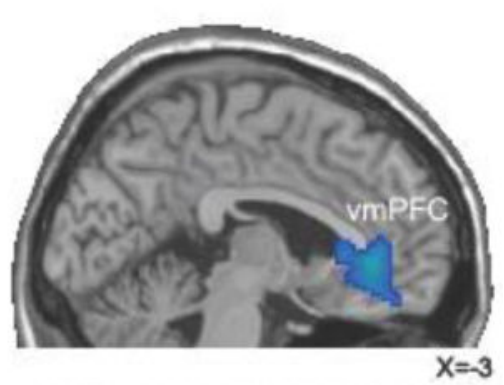

Decreased in Controls compared to A-D (CTRL $<A-D)$

Figure 5.

Between-group differences in brain function associated with the transition to the High Demand state in controls (CTRL), heavy drinkers who were alcohol nondependent (A-ND) and heavy drinkers who were alcohol dependent (A-D). As demand increased from low to high (1- Back to 2-Back), A-D had significantly less activity in the ventral medial prefrontal cortex (vmPFC), compared to CTRL. A-ND had significantly greater activity in the superior parietal cortex (sPar), compared to A-D. Voxel-level probability thresholds were set to $\mathrm{p}<0.01$ and corrected at the cluster level $(F W E, \mathrm{p}<0.05)$. 


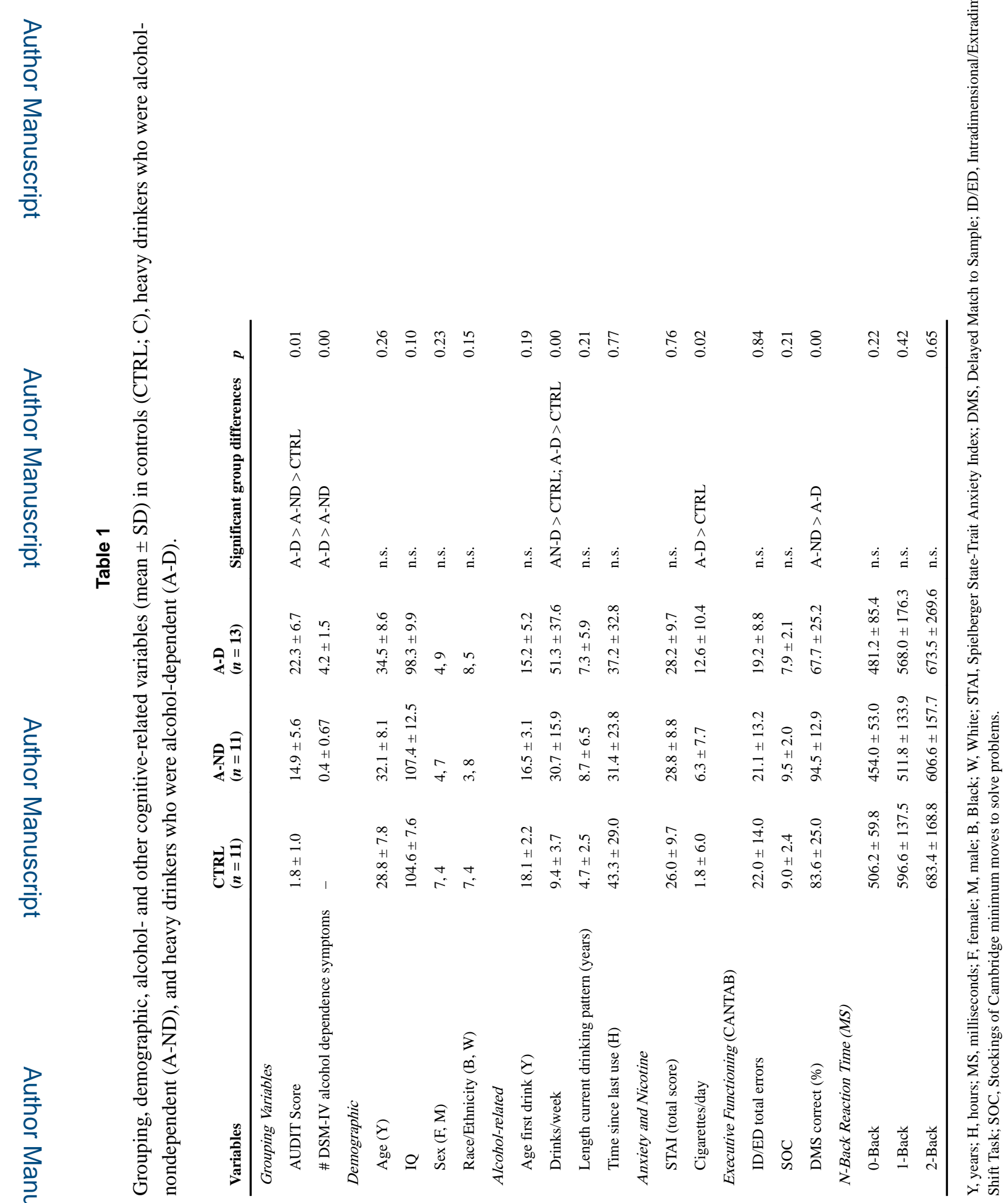




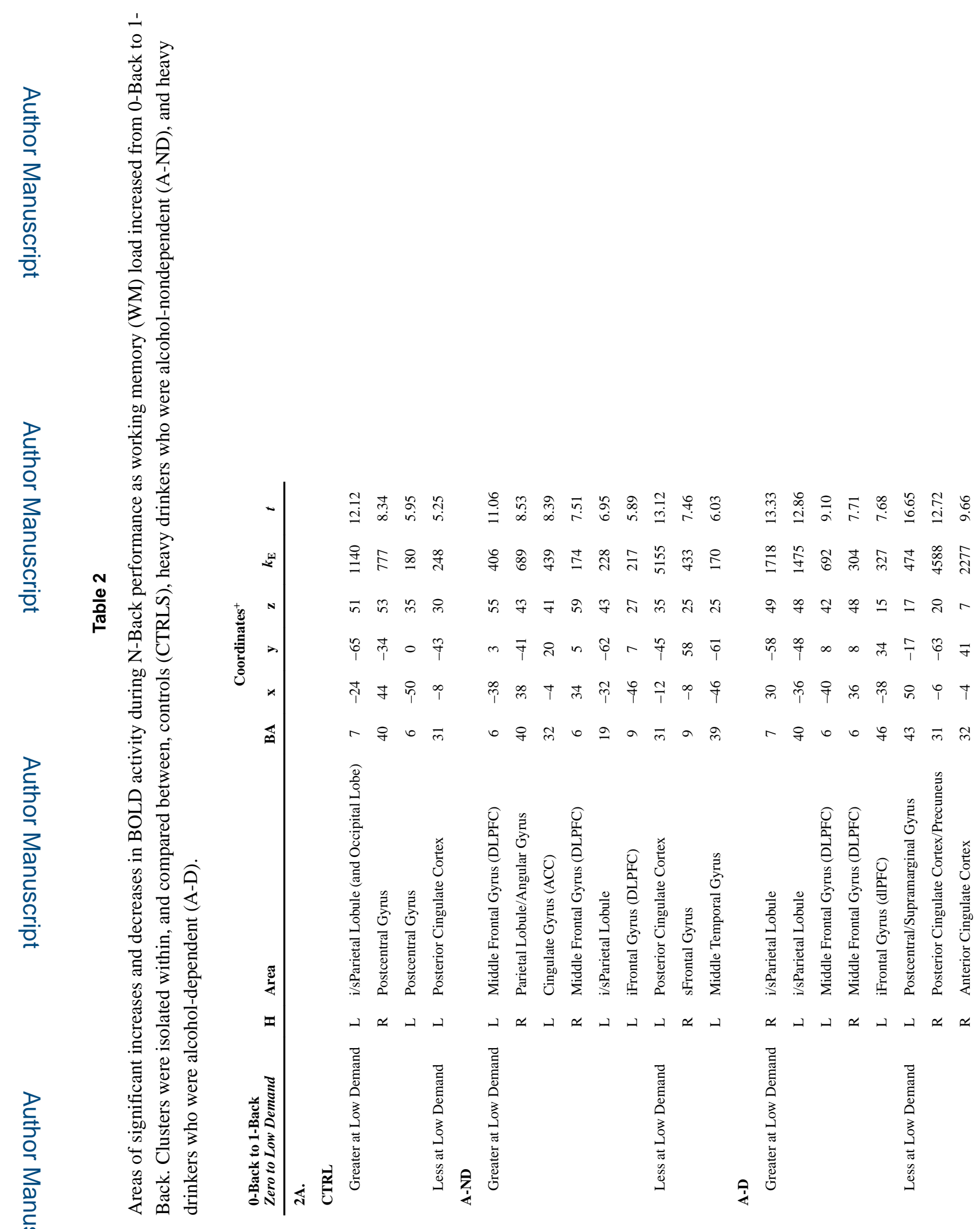




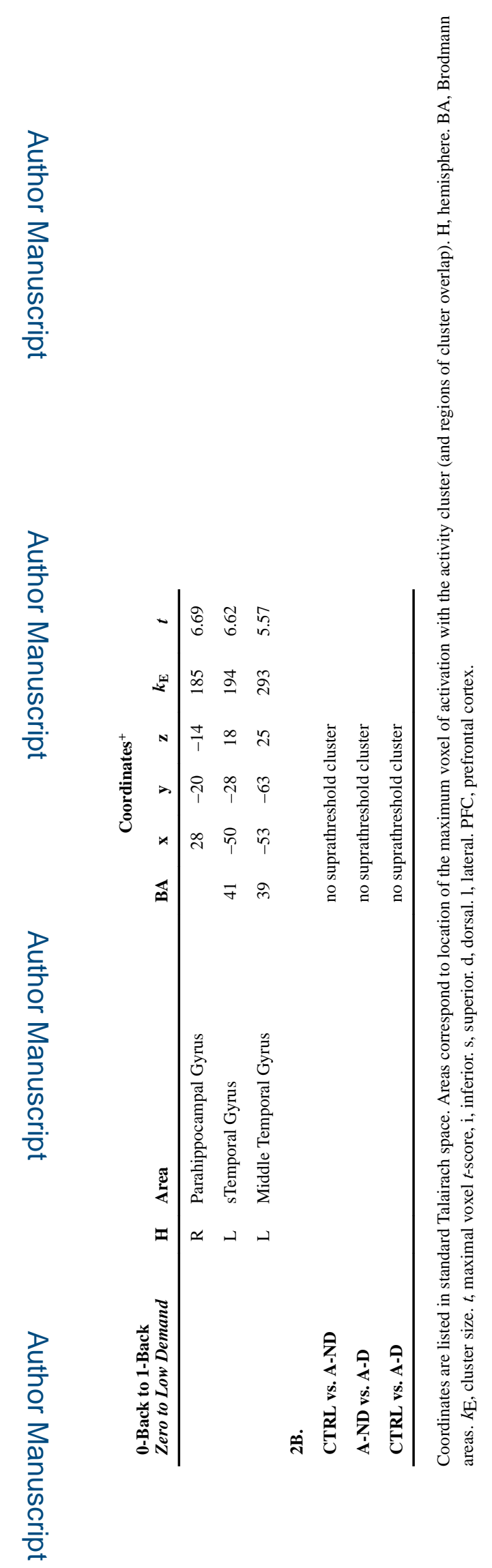

Drug Alcohol Depend. Author manuscript; available in PMC 2018 June 01. 


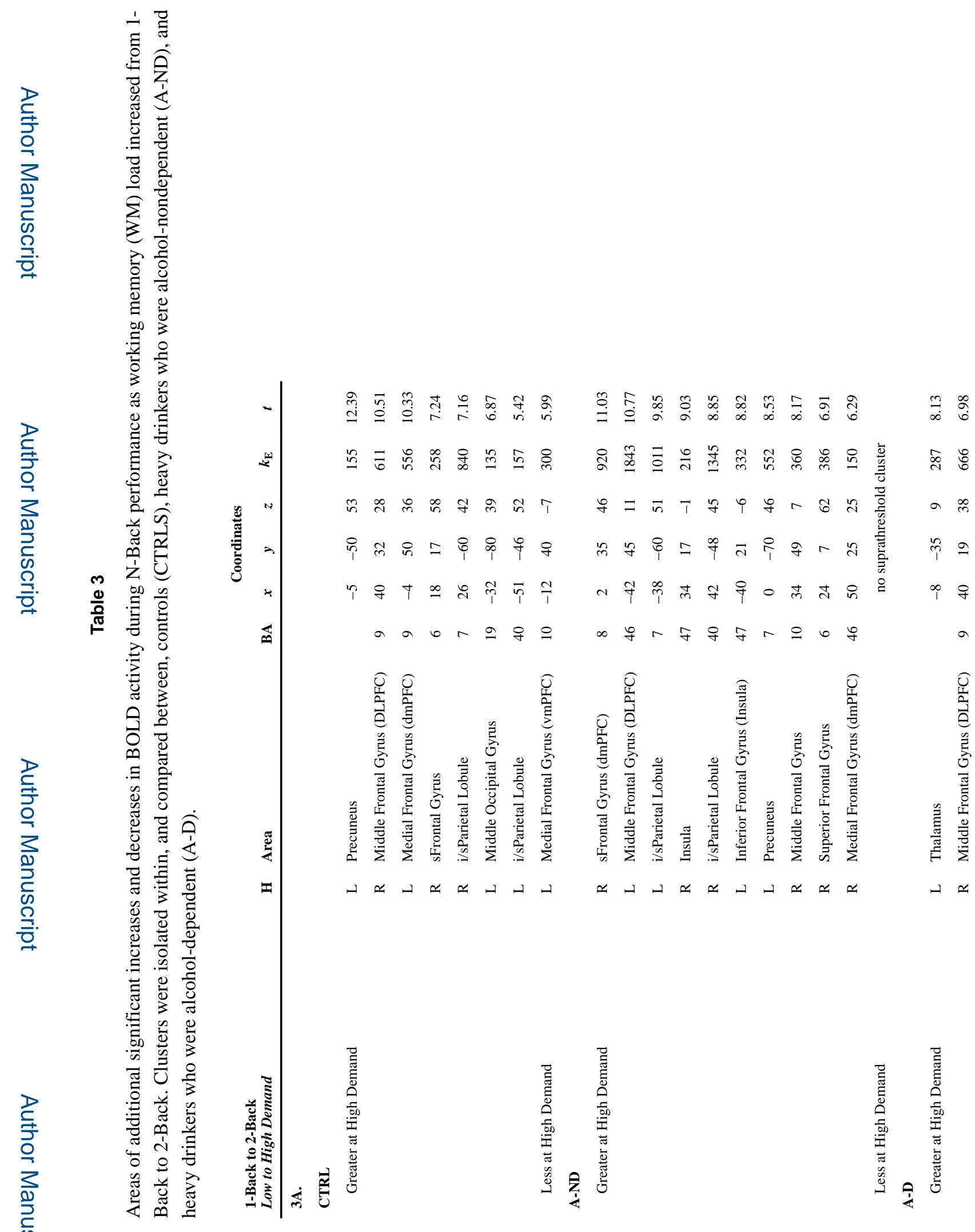




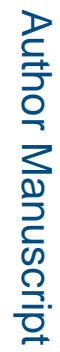

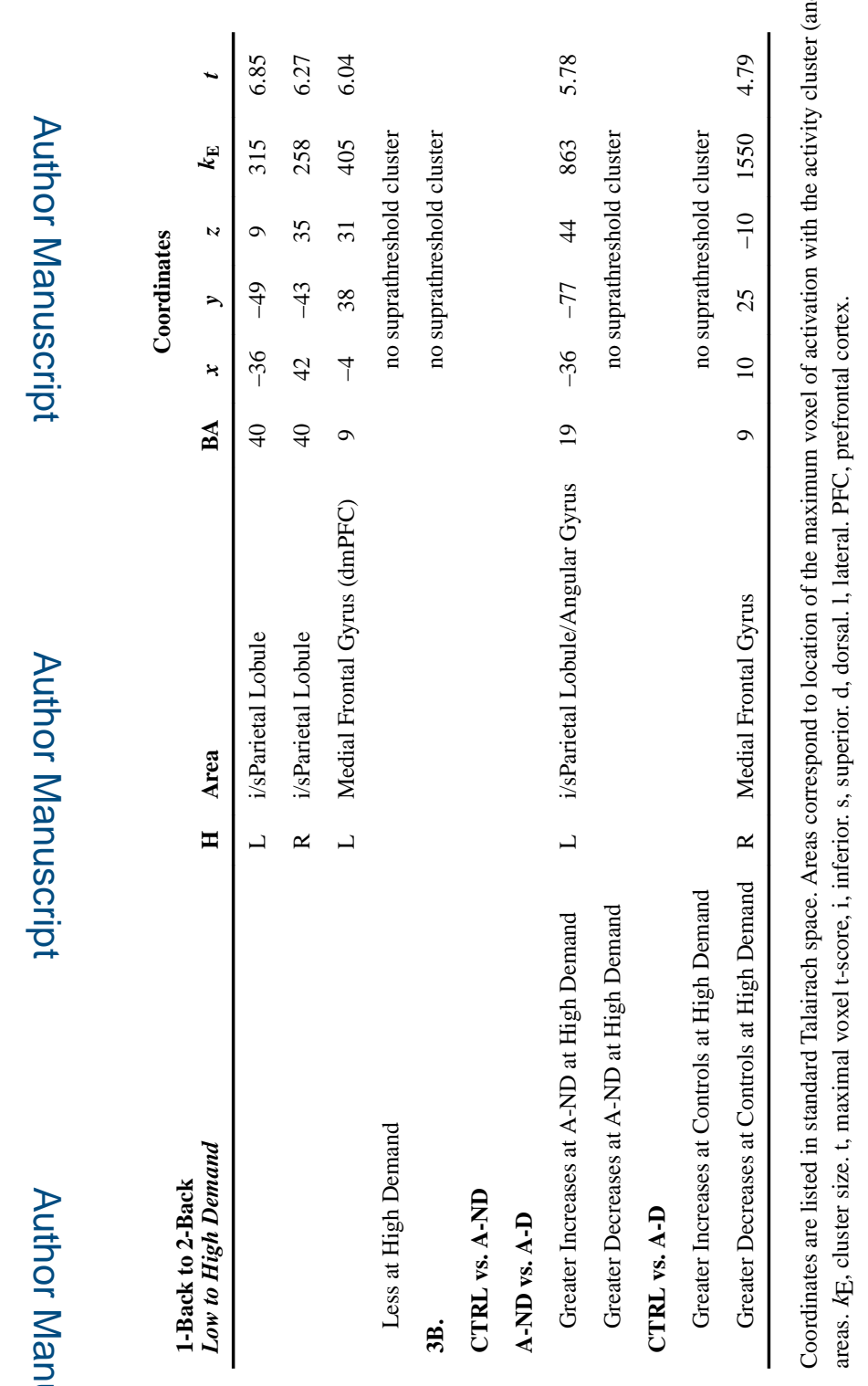

Drug Alcohol Depend. Author manuscript; available in PMC 2018 June 01. 